\title{
Ultrasound of the endometrium: facts, controversies, and future trends
}

\author{
R. L. Bree \\ Department of Radiology, University of Michigan Medical Center, 1500 E. Medical Center Drive, Ann Arbor, MI 48109-0030, USA
}

The role of ultrasound in the evaluation of the endometrium has been overshadowed by a large volume of literature about the ovaries and myometrium. In fact, aside from some investigations centering on the thickness of the endometrium in the normal woman and its relationship to the possibility of endometrial cancer, there has been little interest in the endometrium until very recently. Transvaginal sonography (TVS) has provided a modest improvement over transabdominal sonography (TAS) to image the endometrium and evaluate pathology; however, the gynecologic community relies heavily on endometrial biopsy (EMB), dilatation and curettage (D\&C), and hysteroscopy for endometrial diagnosis.

The introduction of saline-infused hysterosonography (HSG) has revolutionized gynecologic ultrasound of the endometrium. The variety and frequency of endometrial disorders seen with HSG rivals and even surpasses that seen with hysteroscopy, a moderately invasive procedure. This article will attempt to define the role of ultrasound in the complex anatomy, physiology, and pathology of the endometrium.

\section{Techniques}

\section{Transabdominal sonography}

Many sonologists insist on performing TAS as a screening procedure prior to performing any other ultrasound techniques such as TVS or HSG. TAS requires a full bladder and is often uncomfortable and time-consuming, particularly in the postmenopausal woman. For specific evaluation of the endometrium in the patient with a normal-sized uterus, TAS is unnecessary as the first step [1-3]. Furthermore, as the patient becomes well hydrated to fill her bladder for TAS, a partially full bladder interferes with TVS and HSG, which are best performed with the bladder completely empty [4].

When the uterus and ovaries are incompletely visualized on TAS, it may the be appropriate to perform
TAS, but if the uterus is enlarged and anteverted, a full bladder may not be necessary for visualization of the myometrium and ovaries. The uterus can act as an acoustic window.

\section{Transvaginal sonography}

Since its introduction in the mid-1980s, TVS has become the standard way to image the female pelvis in the gynecologic community and an important adjunct to the radiologist/sonologist. End-fire endoluminal probes of 5-9 MHz have been used. The patient, technologist/ sonographer, or sonologist can insert the probe. Imaging is in the coronal or sagittal plane, and the endometrium is well suited for this technique due to its proximity to the vaginal apex, allowing for high resolution scanning [1-5] (Fig. 1).

There is continuing interest in the role of spectral and color Doppler imaging for the endometrium. Either of these techniques may have a role in the differential diagnosis of endometrial abnormalities and are available as adjunctive techniques on most modern ultrasound machines. As with other Doppler applications in the pelvis, there is considerable overlap of benign and malignant abnormalities when measuring indices of resistance, which, when decreased, suggest angioneogenesis associated with neoplasms. Unfortunately, the normal cyclic physiology of the female pelvis produces angioneogenesis in both ovaries and uterus, making these analyses difficult [2]. However, as will be discussed, color Doppler may have a role in differentiating endometrial polypoid lesions.

\section{Hysterosonography}

Although introduced as early as 1986 for potential evaluation of tubal patency, HSG has only recently become popular as an important technique for endometrial evaluation [6-8]. In our department, the procedure is per- 

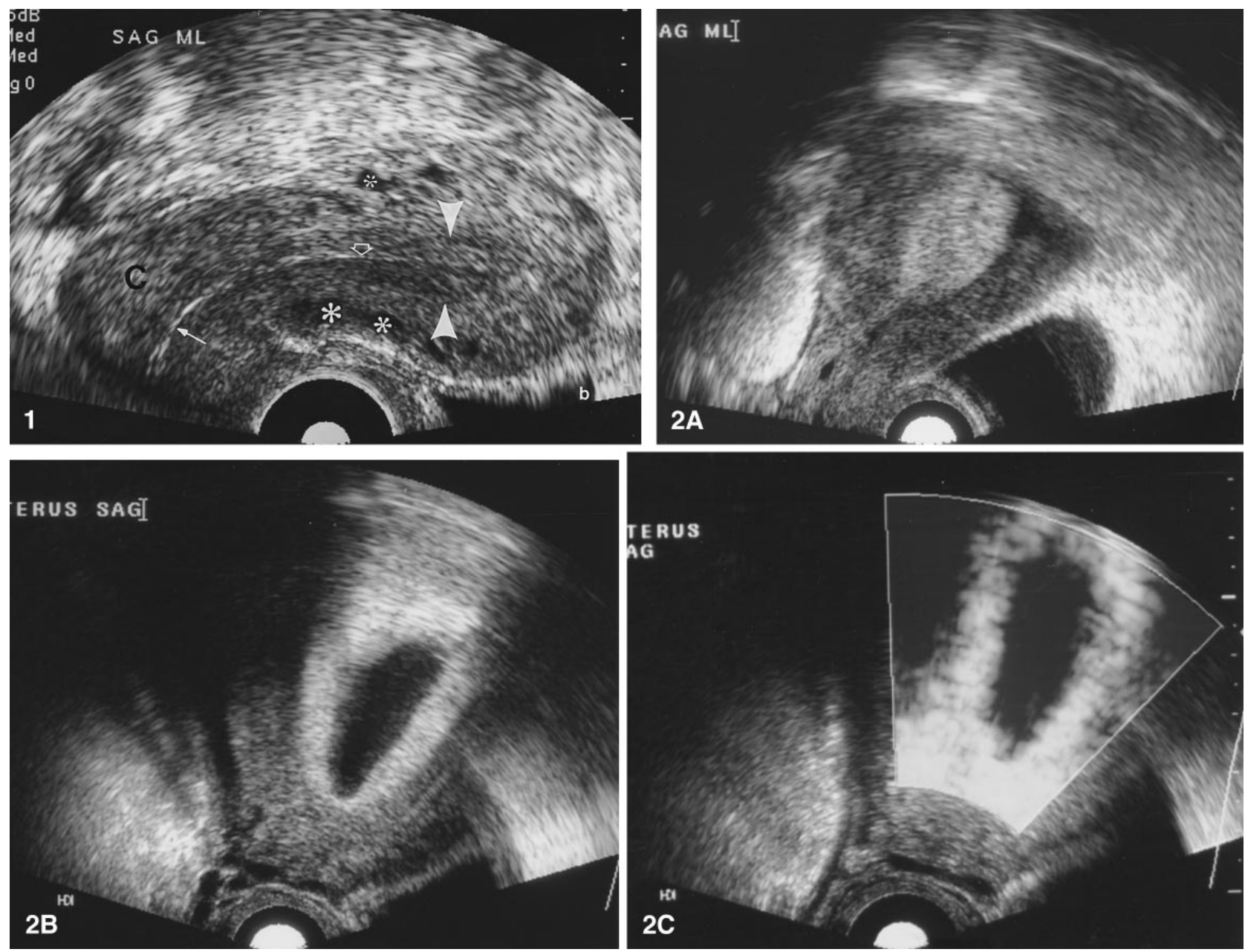

Fig. 1. Transvaginal examination of the normal uterus. A transvaginal scan of the uterus is performed in a sagittal plane. The uterus is anteverted and an almost empty bladder $(b)$ is seen just anterior to the uterine fundus. Moderate prominence of myometrial vessels (*) is seen between the middle and outer zones of the myometrium. The cervix $(C)$ and the endocervical canal (arrow) are clearly visible. The patient is in the proliferative phase, and the endometrium (between arrowheads) is hypoechoic. The endometrial canal (open arrowhead) is clearly visible.

formed following TVS or TAS and TVS, without significant preparation. We use a lighted speculum and a 5-Fr balloon-bearing commercially available HSG catheter (Ackrad Labs, Cranford, NJ).

The patient is placed in the lithotomy position, and the speculum is inserted. The cervix is identified and swabbed with iodine solution. A long cotton swab, available in gynecology departments, is useful. The external cervical os is identified, and the outer stiffener of the catheter system is placed as far into the os as possible. The catheter is then advanced, and the balloon inflated with about $0.5-1 \mathrm{cc}$ of saline, being careful not to instill air, which can create an acoustic artifact. If there is resistance to advancing the catheter, gentle pressure may be applied, and the catheter will often advance

Fig. 2. HSG in premenopausal woman performed at mid-cycle. A Sagittal transvaginal scan demonstrates prominent endometrium compatible with secretory phase. B Following catheterization and installation of fluid, HSG demonstrates normal endometrium with endometrial layers separated by fluid. C Power Doppler image of the endometrium during HSG demonstrates moderate vascularity compatible with secretory endometrium.

over what may be adhesions or areas of stenosis. Rarely, it may be necessary to leave the catheter tip in the cervical canal and inflate the balloon to prevent dislodgment. TAS can be useful to monitor insertion in difficult cases. In very difficult catheterizations, a tenaculum may help stabilize the cervix, or a cervical dilator may open the canal for catheterization. Alternative catheters include urethral balloon catheters, insemination catheters, and pediatric feeding tubes [6-8]. The speculum is then carefully removed without dislodging the catheter. A speculum that disengages or has one open side can facilitate this maneuver.

Following successful placement of the catheter into the endometrial cavity, sterile saline is instilled with transvaginal real-time ultrasound monitoring. Depend- 
ing on the patient's age, uterine morphology, and presence of pathology, we instill 3-40 cc of saline. To prevent leakage of fluid, it is helpful to put traction on the catheter to seal the internal os with the balloon. The catheter should be flushed with saline prior to insertion so no air is instilled. Fluid instillation should be slow to decrease the level of cramping. Some have suggested preparation with ibuprofen to decrease cramping. Images are obtained just after balloon deflation and upon removal of the catheter to image the lower uterine segment free of the catheter artifact. Antibiotics are not routinely given after the procedure [6] (Figs. 2, 3).

In the premenopausal patient, HSG should be performed in the proliferative phase of the menstrual cycle, if possible, to help separate endometrial hyperplasia from secretory endometrium. In the postmenopausal patient, timing of the exam is not as important unless the patient is on cyclic hormonal replacement [7].

\section{Normal anatomy and physiology}

\section{The premenopausal patient}

The endometrium can be seen on TAS, TVS, and HSG. Measurements of the endometrial thickness are best made on TVS or HSG because of the proximity of the transducer to the uterus. The measurement is best made in the sagittal plane, and the convention is to use the full thickness of the endometrium minus any fluid that may be present [2, 3, 8] (Figs. 1, 3A, 4A, 5).

In the normal menstrual cycle, day 1 represents the first day of menstrual flow. A scan performed on that day may show fluid or blood clot in the endometrial cavity (Fig. 5). The endometrium is thinnest on the last day of flow, typically on days 3-5. Endometrial thickness in the proliferative phase of the cycle (days 5-14) is $2-8 \mathrm{~mm}$. In the proliferative phase, the endometrium is hypoechoic, with the lumen maintaining a thin hyperechoic appearance [9, 10] (Fig. 1). Occasionally, the junctional zone between the endometrium and the inner myometrium may appear hypoechoic to the inner zone myometrium and may give the appearance of a halo [11] (Fig. 1). Beginning at day 14, when many women ovulate, the secretory phase begins (Fig. 6). The endometrium becomes thicker, reaching a maximal thickness of $12 \mathrm{~mm}[9,10]$. Furthermore, the endometrium becomes more echogenic due to the change in glandular content from absence of secretions to glycogen and mucin [2, 10] (Figs. 2, 6). The boundary between the endometrium and the inner myometrium again may be hypoechoic and is termed the "junctional zone." The exact histologic correlate of this zone remains controversial, but it is probably a compact inner layer of myometrium [11] (Figs. 1, 4A, 6A).

In the premenopausal patient, HSG can provide additional detail of the endometrial thickness and mor- phology. The addition of color or power Doppler can allow analysis of blood flow and resistance (Fig. 2).

\section{The postmenopausal patient}

Because most pathologic conditions of the endometrium occur during perimenopause and postmenopause, there is much interest in the sonographic appearance in this group of patients. The major clinical problem in this patient group is abnormal bleeding. The amount and significance of the bleeding depends on the presence and type of exogenous hormone replacement therapy (HRT).

Table 1 outlines the major forms of HRT and the expected endometrial thicknesses for patients with and without HRT. In the patient without HRT, an endometrial thickness greater than 5-6 mm may be abnormal, particularly at 5 years postmenopause and beyond, and the asymptomatic patient may require, at minimum, a follow-up examination. In patients with HRT, endometrial thickness may approach that seen during premenopause, particularly for those women on cyclic therapy and those less than 5 years from the onset of menopause. As with the premenopausal population, HSG may be extremely useful for confirmation of normality or for defining pathology [4-8, 12, 13] (Figs. 3, $7,8)$.

\section{Clinical problem solving with ultrasound}

\section{Screening for endometrial cancer}

There is considerable debate in the literature about the value of screening for endometrial cancer in the asymptomatic patient. Osmers et al. [14] argued that a detection rate of $3.5 \%$ when screening asymptomatic women makes screening a worthwhile investment. Unfortunately, like screening for prostate cancer, screening for endometrial cancer has not been subjected to rigorous cost-benefit analysis, taking into account the lead time bias that is present in any screening situation. Is there any data to indicate that an earlier diagnosis of endometrial cancer affects the cure rate and survival? Would not most of these patients eventually present with abnormal uterine bleeding, particularly those in postmenopause [15]?

If screening for endometrial cancer were to be found economically feasible, the data suggest that an endometrial thickness of $4 \mathrm{~mm}$ or less in the asymptomatic postmenopausal patient would exclude all cases of endometrial cancer [14]. In premenopausal patients, there is no role for screening for endometrial cancer. 

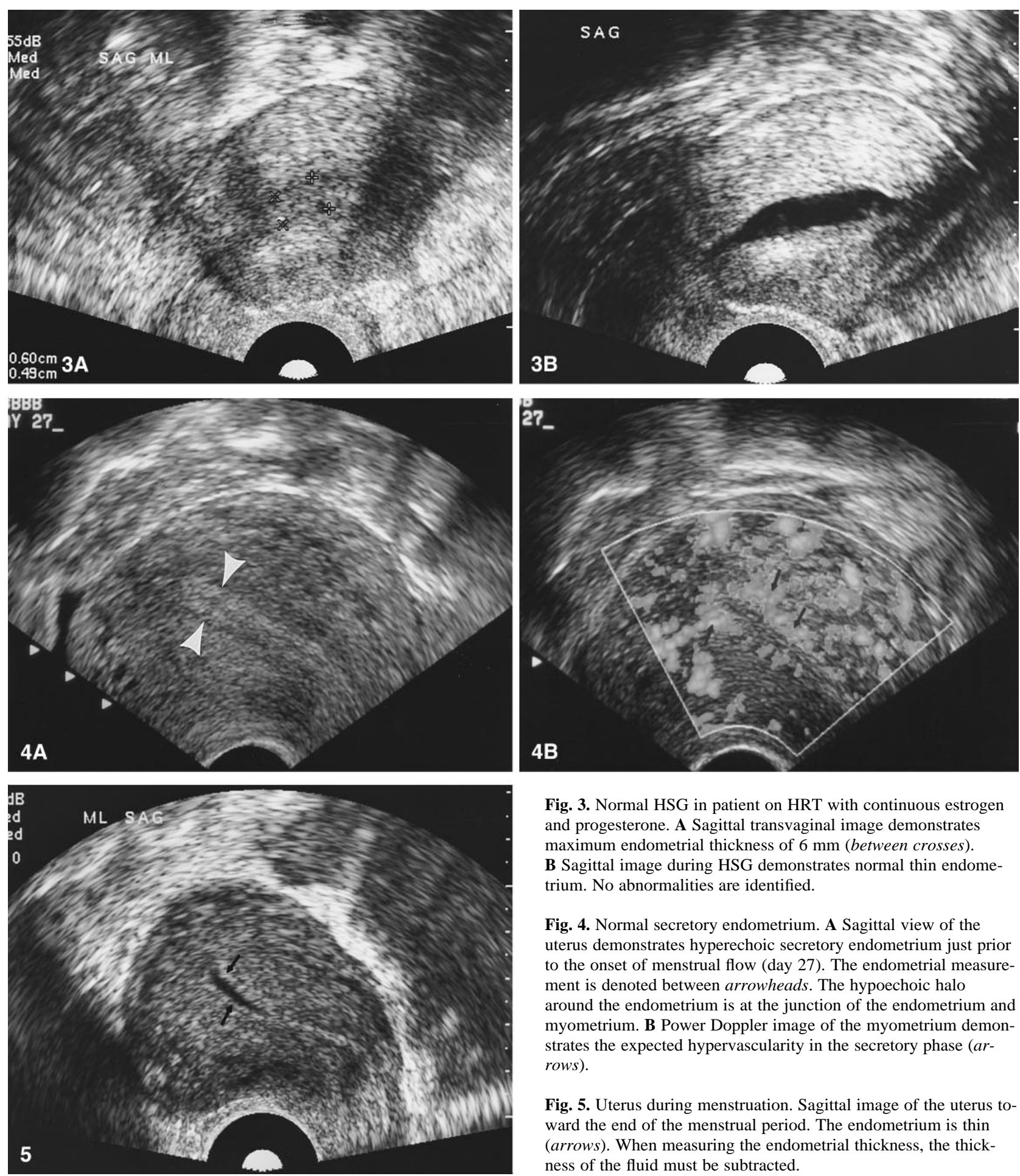

Fig. 3. Normal HSG in patient on HRT with continuous estrogen and progesterone. A Sagittal transvaginal image demonstrates maximum endometrial thickness of $6 \mathrm{~mm}$ (between crosses). B Sagittal image during HSG demonstrates normal thin endometrium. No abnormalities are identified.

Fig. 4. Normal secretory endometrium. A Sagittal view of the uterus demonstrates hyperechoic secretory endometrium just prior to the onset of menstrual flow (day 27). The endometrial measurement is denoted between arrowheads. The hypoechoic halo around the endometrium is at the junction of the endometrium and myometrium. B Power Doppler image of the myometrium demonstrates the expected hypervascularity in the secretory phase ( $a r$ rows).

Fig. 5. Uterus during menstruation. Sagittal image of the uterus toward the end of the menstrual period. The endometrium is thin (arrows). When measuring the endometrial thickness, the thickness of the fluid must be subtracted.

\section{Abnormal uterine bleeding in premenopause}

It is difficult to define the exact cause of abnormal or irregular uterine bleeding (AUB) in the premenopausal patient, but pathologic conditions of the endometrium or myometrium often are factors. Anovulatory cycles

are a common cause, but hyperplasia, polyps, submucous myomas, and carcinoma are possible and of concern to the patient. Clinical procedures often used to evaluate patients with AUB include D\&C and endometrial biopsy (EMB), with hysteroscopy reserved for complex cases with persistent bleeding [16-18]. Pa- 

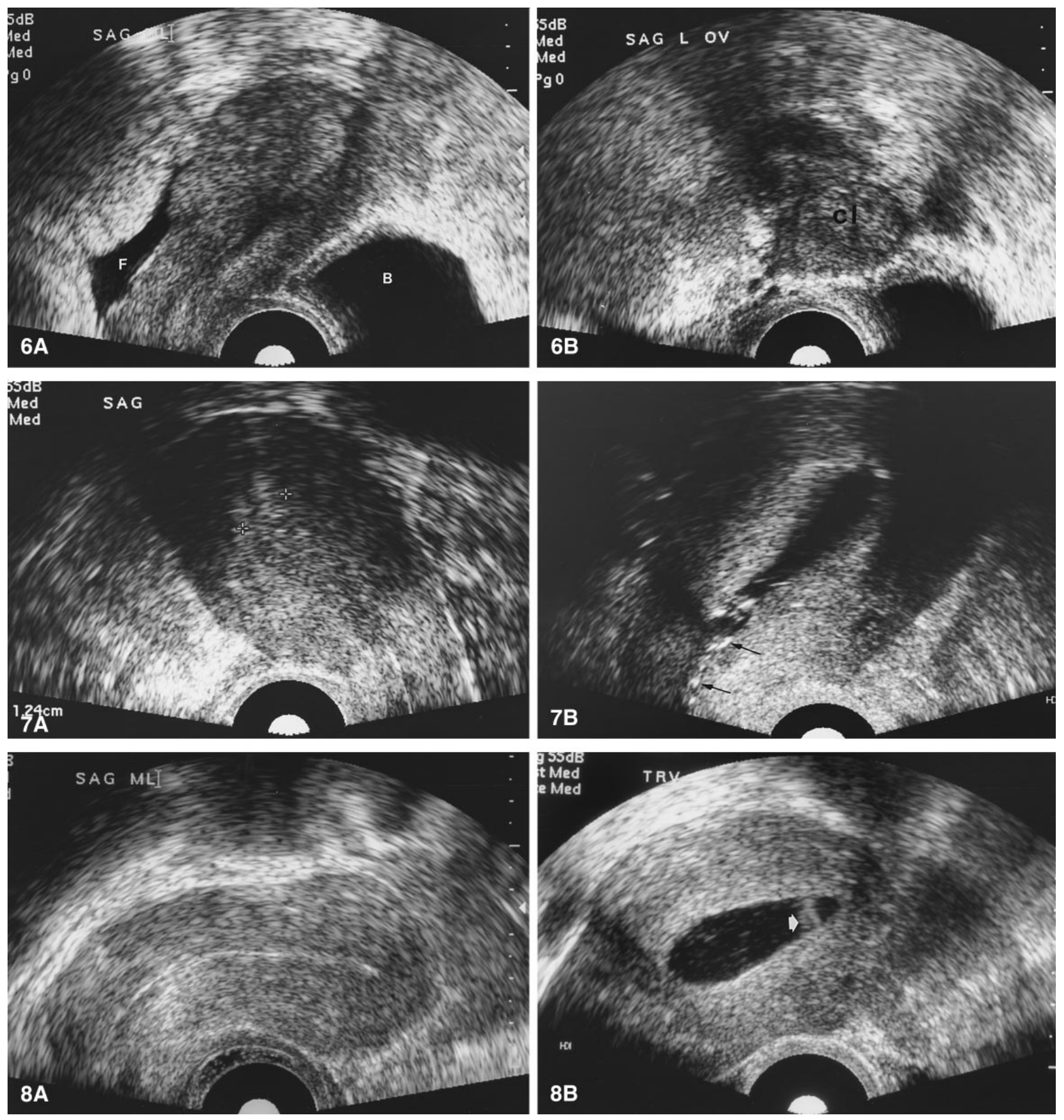

Fig. 6. Recent ovulation and corpus luteum formation. A Sagittal image through the uterus demonstrates partially filled bladder $(B)$ anteriorly and free intraperitoneal fluid posteriorly $(F)$ due to recent ovulation. The endometrium is hyperechoic, indicating early secretory phase. B View of the left ovary demonstrates hyperechoic corpus luteum $(\mathrm{cl})$ due to intense hemorrhage following ovulation.

Fig. 7. Normal secretory endometrium in postmenopausal patient on cyclic HRT. A Transvaginal scan in the sagittal plane demonstrates mildly thickened endometrium $(12.4 \mathrm{~mm})$ identified between the

curser marks. Endometrial measurement was difficult in this patient. B HSG demonstrates mildly prominent endometrium without other pathology. D\&C demonstrated secretory endometrium. Echoes from the catheter are seen in the lower uterine segment (arrows).

Fig. 8. Endometrial polyp with normal endometrium on TVS. A Postmenopausal patient with bleeding. TVS demonstrates very thin endometrium. B HSG demonstrates small polyp (arrowhead) not visible on TVS. Multiple echoes in the instilled fluid are air bubbles. 
Table 1. Postmenopausal endometrial thickness (asymptomatic patient)

\begin{tabular}{lc}
\hline Normal hormone status & Maximum thickness (mm) \\
\hline No hormonal replacement & 6 \\
Unopposed estrogen & 8 \\
Continuous estrogen + progesterone & 8 \\
Sequential estrogen + progesterone & $15^{a}$ \\
& $8^{b}$
\end{tabular}

${ }^{a}$ In secretory phase

${ }^{b}$ In menstrual or proliferative phase

tients with menorrhagia fall into a similar diagnostic dilemma as those with AUB because submucous leiomyomas and polyps are causes, as are generalized leiomyomas with uterine enlargement and adenomyosis.

The value of ultrasound in evaluating and triaging these patients cannot be overemphasized. TAS may be indicated in the patient with an enlarged uterus at the time of physical examination, but TVS and HSG are critical in the overall evaluation and clinical care of patients with abnormal bleeding. Although an endometrial diameter of $4 \mathrm{~mm}$ or less excludes most pathology, our experience suggests that HSG is useful even in patients with thin endometria (Fig. 8). When HSG is performed in premenopausal patients, it is best done in the proliferative phase of the cycle. The sonologist should carefully evaluate the endometrial texture to search for small filling defects that can be better evaluated with HSG [16, 19, 20] (Fig. 9).

Although leiomyomata can often be detected and followed into the endometrium with TVS, HSG provides a clearer evaluation of the size of the mass and can help assess whether the patient is a candidate for an hysteroscopic myomectomy. If the leiomyoma is intracavitary or predominantly submucous, hysteroscopic resection is feasible (Fig. 10). If the lesion is predominantly mural, hysterectomy is the only choice if the AUB can be attributed to the leiomyoma [16, 17, 21]. Cicinelli et al. [22] suggested a transabdominal approach to HSG imaging when evaluating leiomyomata.

Another cause of AUB is endometrial hyperplasia. It is most commonly cystic, but the cysts are microscopic. Adenomatous hyperplasia, the other form, is sonographically identical in appearance. With TAS, TVS, and HSG, there is uniform increased endometrial echogenicity that can also be hypervascular with color Doppler [1, 2, 7, 17, 23].

Merce et al. [24] described the role of spectral Doppler in the evaluation of the endometrium in patients with AUB. As with other Doppler evaluations attempting to separate benign from malignant histologies, the positive predictive value is poor and the information does not influence the eventual diagnostic or therapeutic outcome. Others have used uterine artery waveforms to assess endometrial pathology and have found some suc- cess in the evaluation of the resistive index in separating cancer from benign disorders [25]. We have not used this technique and feel as Granberg and Bourne [4, 15] that it does not add significantly to the specificity obtained with HSG or change clinical management of the patient.

\section{Postmenopausal bleeding}

Any postmenopausal patient who is not scheduled to bleed from HRT suffers from postmenopausal bleeding (PMB) and should have a diagnostic evaluation. The obvious fear in this patient population is endometrial cancer. In the past, diagnostic tests such as EMB, $\mathrm{D} \& \mathrm{C}$, hysteroscopy, and even hysterectomy were used to allay the fear of cancer. Even though endometrial atrophy is the most common cause of PMB and cancer is only seen in about $10 \%$ of patients with PMB, defining the absence of significant pathology is important in this patient population [26]. Finding less significant but treatable abnormalities, such as an endometrial polyp, is important for the psychological health of the patient. In addition, endometrial polyps may be associated with or progress to endometrial cancer, and polypectomy accompanied by $\mathrm{D} \& \mathrm{C}$ may be indicated in these patients [27].

Ultrasound has a definite role in the evaluation of patients with PMB. Conventional diagnostic procedures have significant false-negative rates for the common conditions such as polyp, leiomyoma, and cancer [15]. Even though hysteroscopy performed in the office can be cost-effective in defining the etiology of PMB, polypectomy and directed biopsy is difficult with this instrument and most gynecologists are reluctant to perform this uncomfortable procedure in an office setting.

We have begun to study every patient with PMB with TVS followed by HSG. Our experience has been that using TVS alone cannot define all of the abnormalities and that normal thickness endometria can harbor polyps or submucous leiomyomata (Figs. 8, 9, 11). Furthermore, defining a thickened endometrium does little to determine the etiology, and many polyps appear as a thickened endometrium at first glance (Fig. 12). However, in the multicenter "Nordic Trial," 35\% of women with PMB with endometrial thicknesses of $6-$ $10 \mathrm{~mm}$ had atrophic endometria histologically [28] (Figs. 3, 7).

Several features favor an endometrial polyp with TVS and confirm the presence of a polyp with HSG. Cystic spaces in the endometrium are characteristic of polyps [29-32]. The spaces may represent dilated glands seen histologically (Fig. 13). Polyps tend to be on a thin stalk and with color Doppler have a single vessel entering the stalk [6] (Figs. 8, 9, 12). The vascularity of a polyp is less than that of a pedunculated submucous leiomyoma, which can be mistaken for a 

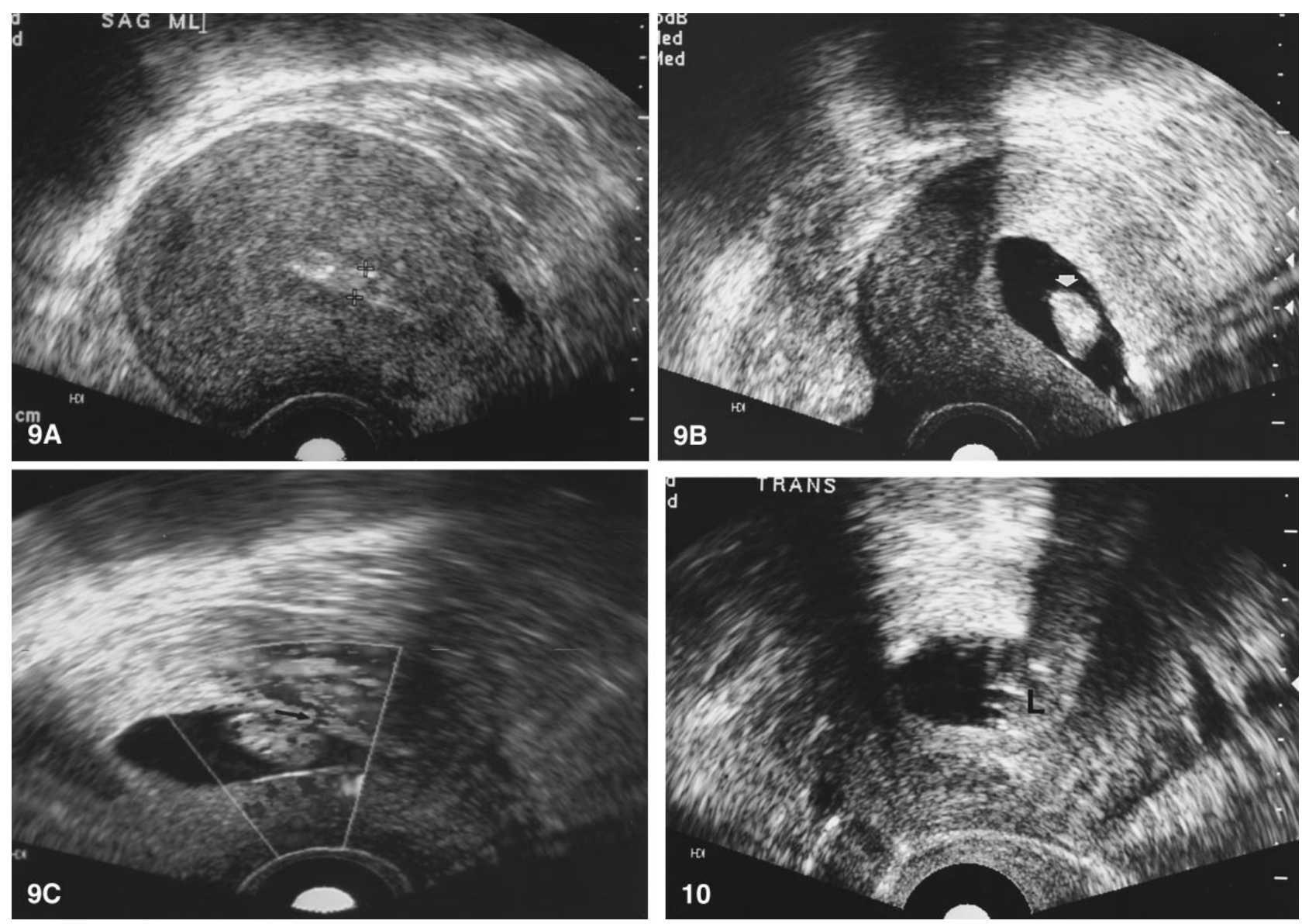

Fig. 9. Perimenopausal patient with endometrial polyp. A Sagittal TVS demonstrates mild uterine enlargement with $5 \mathrm{~mm}$ endometrium and heterogenous echo pattern, suggesting a polyp. B Gray-scale image during HSG demonstrates endometrial polyp (arrow) with otherwise normal endometrium. C Power Doppler image demonstrates single vessel entering the stalk of the polyp (arrow), a common feature of endometrial polyps.

polyp with HSG (Fig. 14). Polyps may be multiple, and careful search for others is necessary when a large polyp is initially discovered (Fig. 12). Polyps are typically echogenic with TVS and with HSG, although they are occasionally hypoechoic with TVS. The increased echogenicity with HSG is related to the differential echodensity with the surrounding fluid and to acoustic enhancement (Fig. 9).

Submucous leiomyomata are a cause of PMB. Leiomyomata are often inhomogeneous with mixed echogenicity, and they disrupt the endometrium $[6,7]$. When a leiomyoma is large and has continuity with the myometrium, the distinction from a polyp or carcinoma is easy (Figs. 10, 14). Occasionally, a submucous leiomyoma is pedunculated and mimics the appearance of a polyp. In this instance, differentiation is more difficult (Figs. 11, 15). Features suggesting leiomyoma include a broader base, irregular shape, and increased vascular-
Fig. 10. Perimenopausal woman with abnormal uterine bleeding and submucous leiomyoma. Image in the transverse plane from HSG demonstrates large submucous leiomyoma $(L)$, filling almost $50 \%$ of the endometrial cavity at this level.

ity with color Doppler (Figs. 11, 14, 15). Our experience suggests that the pedunculated polyplike leiomyomata are more commonly the cause of PMB than the larger lesions connected to the myometrium.

As in the premenopausal woman, detection and staging of submucous leiomyomata can be useful in the preoperative decision of hysteroscopic versus open treatment of the lesion depending on the size and percentage of protrusion into the endometrial cavity. HSG is an ideal procedure to provide that information $[21,22]$.

Although not a cause of bleeding, uterine adhesions or synechiae have been discovered in patients with PMB. Adhesions are probably the result of previous uterine instrumentation and are associated with infertility in premenopausal women [6, 7] (Fig. 16). In addition, linear bands may be remnants of hemorrhage and only represent fibrin strands when they are seen shortly 

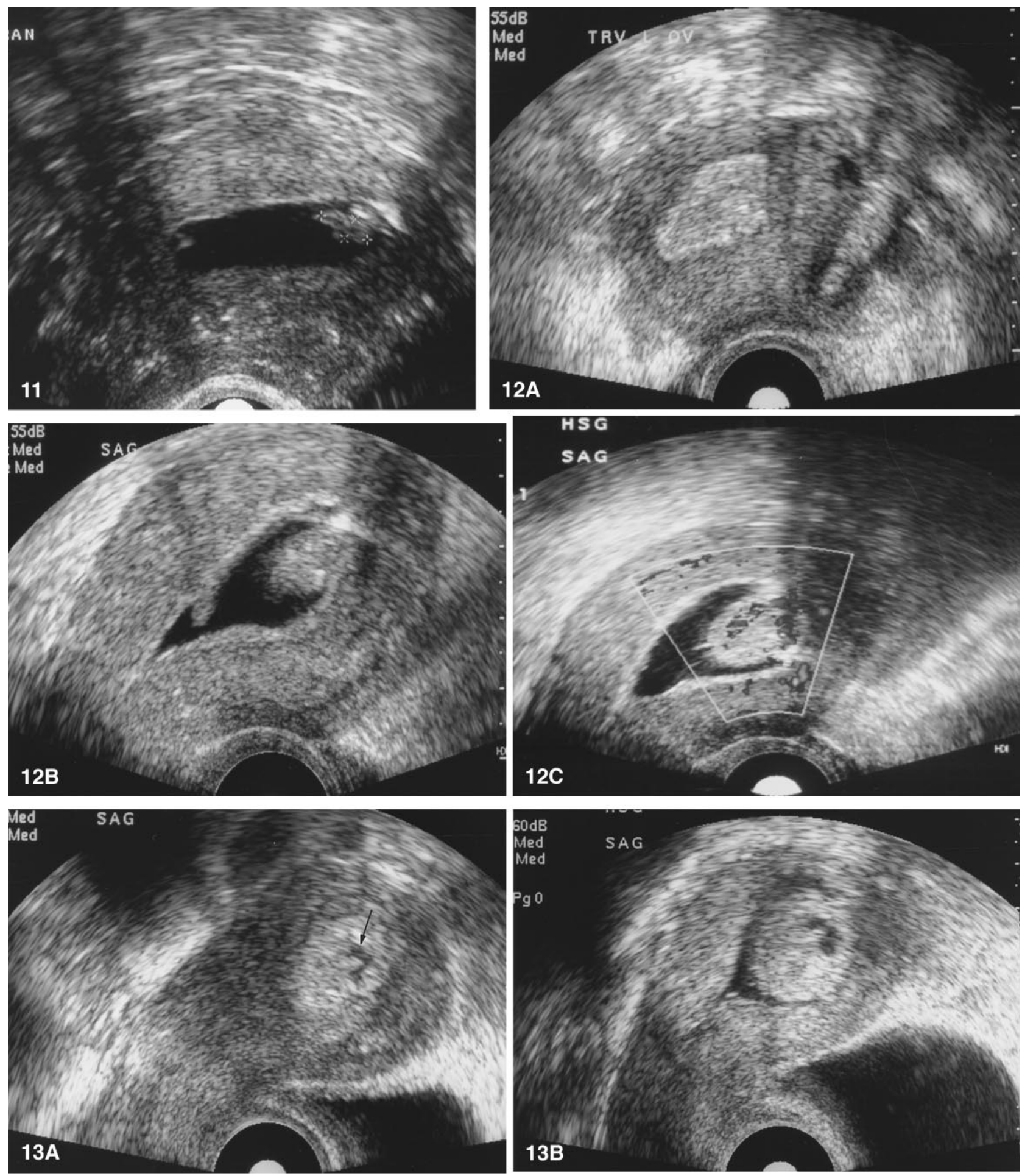

Fig. 11. Polypoid submucous leiomyoma. A patient with postmenopausal bleeding. An initial TVS showed a thin endometrium. HSG demonstrates small irregular polypoid mass subsequently proven to be submucous leiomyoma. These can be extremely difficult to separate from denomatous polyps.

Fig. 12. Endometrial polyp mimicking thickened endometrium. A Patient with postmenopausal bleeding demonstrates thickened endometrium on TVS. B HSG demonstrates one large and one small polyp with normal endometrium. The apparent thickened endome-

trium was the large polyp. C Power Doppler image demonstrates relatively hypovascular appearance of the polyp with a single vessel emanating from the stalk.

Fig. 13. Tamoxifen induced polyp with cystic spaces. A TVS demonstrates enlarged hyperechoic endometrium with central hypoechoic region (arrow). B Sagittal image during HSG illustrates the presence of a large polyp causing the apparent thickened endometrium. The hypoechoic region is a cystic space. 

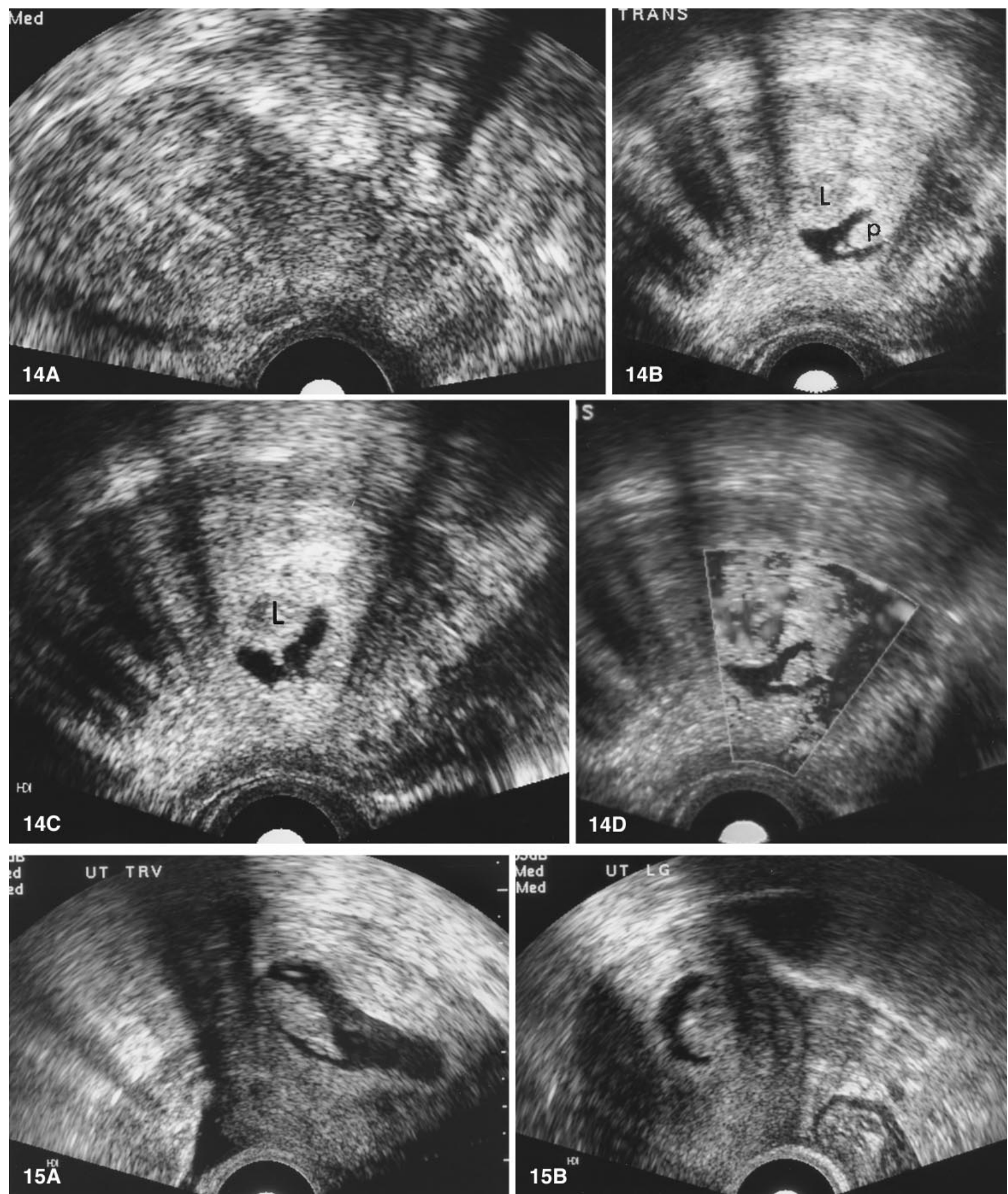

Fig. 14. Endometrial polyp and submucous leiomyoma in postmenopausal bleeder. A TVS shows poor visualization of the endometrium, which is irregular and inhomogeneous. B Transverse image of HSG demonstrates well-defined polyp $(p)$ with narrow stalk. The edge of a leiomyoma $(L)$ is seen on the same image. $\mathbf{C}$ Transverse image through a different plane demonstrates hypoechoic leiomyoma $(L)$ protruding into the endometrial cavity. D Power Doppler image demonstrates polyp and leiomyoma. The polyp is hypovascular and the leiomyoma is hypervascular.

Fig. 15. Submucous leiomyoma mimicking polyp. A Patient with postmenopausal bleeding and largely intraluminal filling defect looking like a polyp on the transverse plane. B Sagittal image demonstrates broader base to the intraluminal mass, with apparent continuity from the myometrium, suggesting a leiomyoma. 

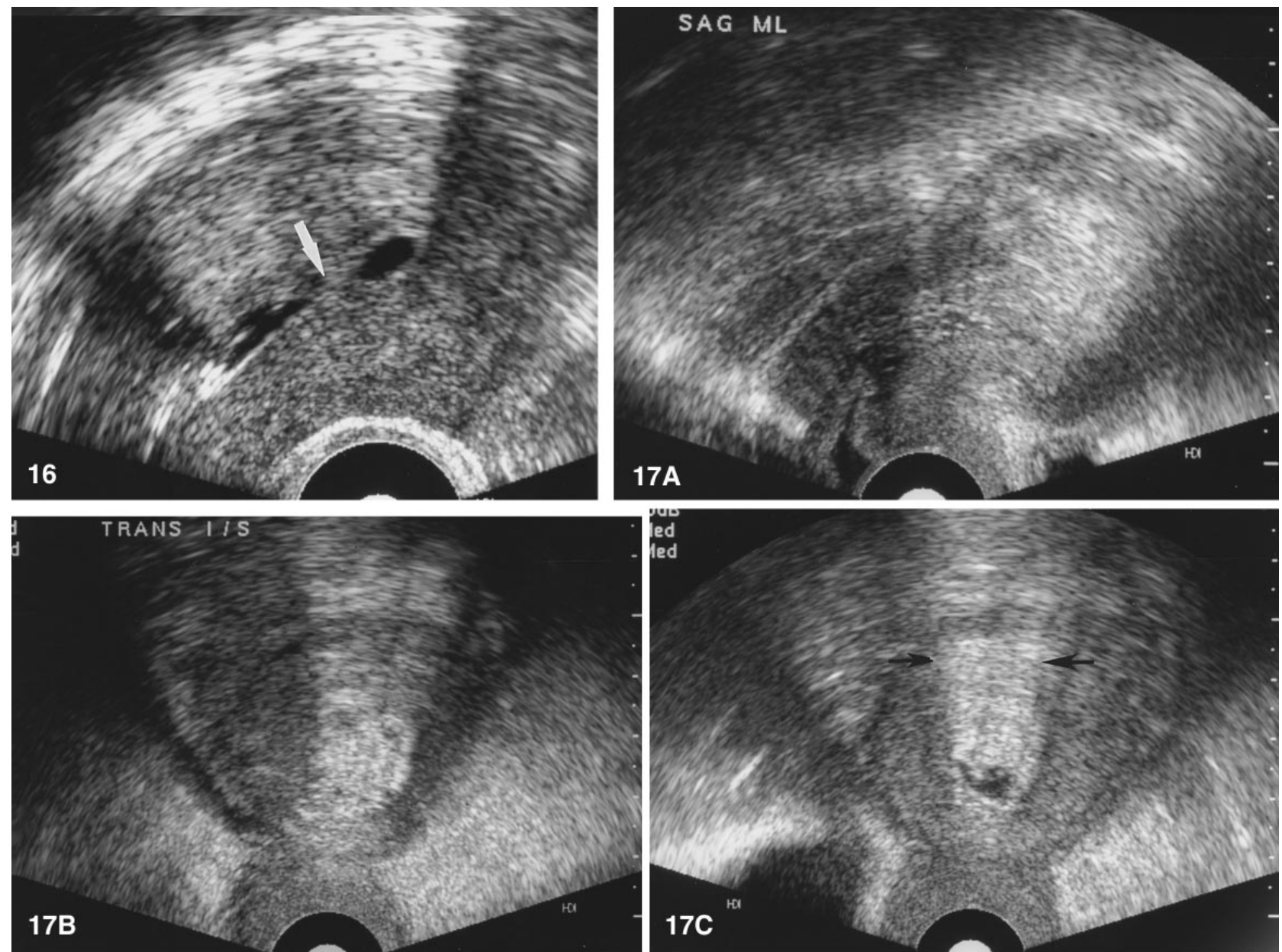
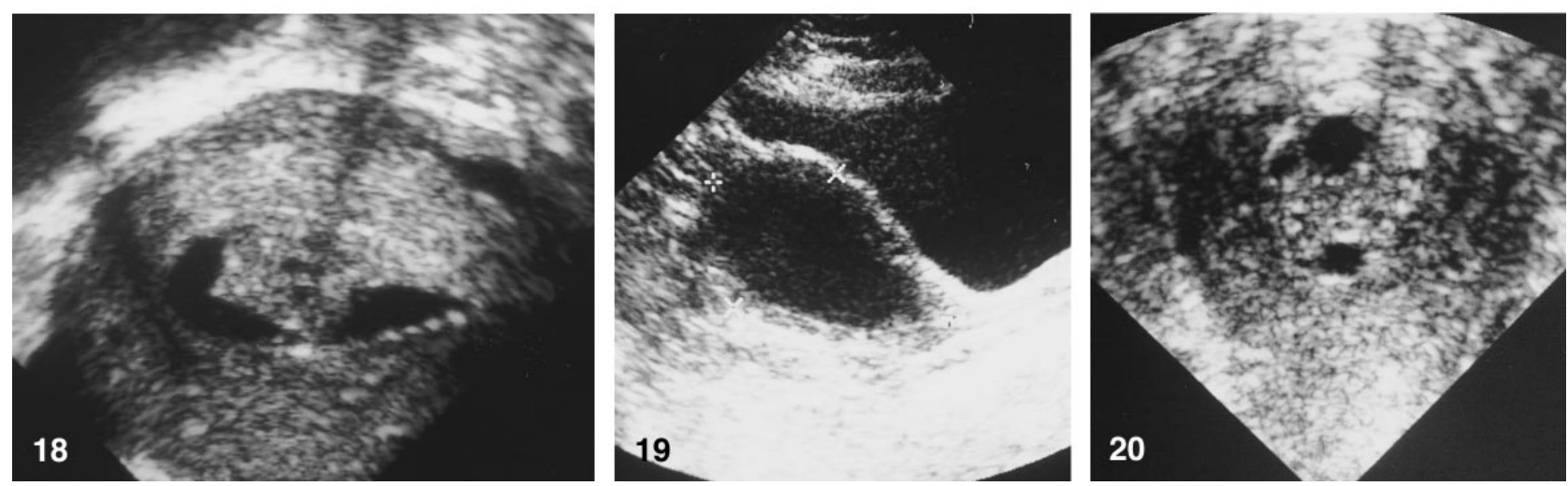

Fig. 16. Uterine synechia. Bright linear echo seen during HSG on patient with postmenopausal bleeding is a synechia (arrow). The patient had had no recent instrumentation, making a fibrin strand unlikely.

Fig. 17. Endometrial cancer in patient with postmenopausal bleeding. A Sagittal image from TVS shows poorly defined thickened endometrium with inhomogeneity. B Transverse image from TVS shows very thick endometrium, with inhomogeneity consistent with endometrial cancer. C Transverse image during HSG demonstrates poor filling of the endometrial cavity due to large endometrial mass. The carcinoma extends deep into the myometrium (arrows).

Fig. 18. Endometrial cancer. A broad-based polypoid mass is seen protruding into the endometrial cavity. This mass proved to be an endometrial cancer. (Courtesy of Marcella Bohm-Velez, Pittsburgh, PA)

Fig. 19. Pyometra. Transabdominal ultrasound performed on postmenopausal patient with vague pelvic pain demonstrated large fluid collection filling the endometrium with very thin myometrium, which proved to be a pyometra secondary to cervical stenosis.

Fig. 20. Tamoxifen changes in the subendometrium. Breast cancer patient with long-standing tamoxifen therapy. Postmenopausal bleeding was evaluated with ultrasound, demonstrating cystic changes. HSG was not performed. D\&C yielded scant atrophic tissue. Cystic changes are presumed due to subendometrial adenomyosis. 
after EMB, a common scenario for this patient population. To decrease the incidence of false-positive synechiae, we usually wait at least 1 week after EMB prior to performance of HSG.

Exclusion of endometrial cancer is the goal of evaluating the patient with PMB. As a result, evaluation with ultrasound, Doppler, or HSG is only ancillary to a definitive biopsy either in the office or in the operating room [33-36]. However, TVS demonstration of an endometrial thickness of $4 \mathrm{~mm}$ or less virtually excludes carcinoma with the same reliability as an EMB plus D\&C. When TVS demonstrates a thin endometrium and the question asked is strictly related to presence or absence of cancer, biopsy is not necessary; in the Nordic Trial, the number of biopsies was decreased by $46 \%$ without loss of accuracy [28]. If an EMB is inadequate, as happens frequently, TVS with or without HSG can be used to determine if the inadequate tissue sample is due to endometrial atrophy or to a technical failure requiring further evaluation with D\&C and hysteroscopy. In cases where the bleeding is sufficiently distressing to the patient, HSG should be done, with definitive therapy planned accordingly.

The appearance of endometrial cancer with TVS and HSG is well described. With TVS, the endometrium is diffusely thickened, irregular, and echogenic [1, 2] (Fig. 17). Uncommonly, endometrial cancer is a broad-based polypoid mass [1] (Fig. 18). Ultrasound has been advocated for assessing myometrial invasion by endometrial cancer [31, 32, 37-39]. Superficial invasion is associated with the preservation of the subendometrial halo, whereas deep invasion is associated with loss of the halo [38].

\section{Endometrial fluid}

The incidental finding of fluid in the endometrial cavity is less ominous during premenopause than during postmenopause. In the presence of bleeding, fluid can often be seen. In the absence of bleeding during postmenopause, a fluid collection is often the result of cervical stenosis and can be due to any of the previously described etiologies of bleeding, although some series have suggested a very low incidence of cancer $[4,32$, 40]. Unfortunately, it is often impossible to perform an EMB or even to perform D\&C and hysteroscopy without a major surgical procedure to dilate the cervix.

One of the complications of long-standing fluid in the endometrial cavity is infection leading to pyometra (Fig. 19), which often leads to hysterectomy. Ultrasound can be useful to discover the etiology of the fluid and to guide surgical or radiologic aspiration of the fluid.

\section{Tamoxifen-related abnormalities}

Tamoxifen is a commonly used drug for adjuvant treatment of breast cancer in postmenopausal women and is also being investigated for prophylaxis against breast cancer. The hormone has estrogenic and antiestrogenic effects and is the cause of endometrial and subendometrial abnormalities. Bleeding is common, but abnormalities are seen in the absence of bleeding. Screening for tamoxifen changes in the uterus may be indicated [41].

Tamoxifen can lead to endometrial hyperplasia, carcinoma, and cystic subendometrial changes, which may be due to adenomyosis [42-44]. Tamoxifen is associated with an increased incidence of endometrial polyps; as a consequence, when cystic changes are identified in a thickened endometrium in patients on tamoxifen, HSG is necessary to define the pathology and guide further diagnosis and therapy [45-47]. When a polyp is identified, hysteroscopic removal is indicated (Fig. 13). When hyperplasia or carcinoma is suggested, EMB or $\mathrm{D} \& \mathrm{C}$ should be done. If the changes are all subendometrial, no biopsy is necessary (Fig. 20).

\section{Conclusions}

Modern gynecologic ultrasound has revolutionized the evaluation of women with common endometrial-related problems previously relegated to blind and inaccurate diagnostic procedures. The development and increasing popularity of HSG has led to increased understanding of the endometrium seen with TVS. It is now possible for the experienced sonologist to examine the endometrium with TVS and predict normality or abnormality and identify polyps, leiomyomata, or carcinoma. This possibility does not preclude performance of $\mathrm{HSG}$, a safe, effective, and simple procedure, but it does provide for greater confidence in the results of TVS when HSG is contraindicated or not possible because of cervical stenosis.

With high-resolution TVS and HSG, we have developed an understanding of the normal endometrium and the normal variations with the various forms of HRT. In the presence of abnormal uterine bleeding, TVS and HSG will provide important information for accurate diagnosis and therapy in a large population of patients.

\section{References}

1. Atri M, Nazarnia S, Aldis AE, et al. Transvaginal US appearance of endometrial abnormalities. RadioGraphics 1994;14:483-492

2. Mogavero G, Sheth S, Hamper UM. Endovaginal sonography of the nongravid uterus. RadioGraphics 1993;13:969-981

3. Lewit N, Thaler I, Rottem S. The uterus: a new look with transvaginal sonography. J Clin Ultrasound 1990;18:331-336

4. Granberg S, Bourne TH. Transvaginal ultrasonography of endometrial disorders in postmenopausal women. Ultrasound $Q$ $1995 ; 13: 61-74$ 
5. Varner RE, Sparks JM, Cameron DC, et al. Transvaginal sonography of the endometrium in postmenopausal women. Obstet Gynecol 1991;78:195-199

6. Cullinan JA, Fleischer AC, Kepple DM, et al. Sonohysterography: a technique for endometrial evaluation. RadioGraphics 1995; 15:501-514

7. Parsons AK, Lense JJ. Sonohysterography for endometrial abnormalities: preliminary results. J Clin Ultrasound 1993;21:8795

8. Wolman I, Jaffa AJ, Hartoov J, et al. Sensitivity and specificity of sonohysterography for the evaluation of sonohysterography for the evaluation of the uterine cavity in perimenopausal patients. $J$ Ultrasound Med 1996;15:285-288

9. Santolaya-Forgas J. Physiology of the menstrual cycle by ultrasonography. J Ultrasound Med 1992;11:139-142

10. Eden JA, Place J, Carter GD, et al. What are the ultrasound and biochemical features of impending ovulation? Aust NZ J Obstet Gynaecol 1988;28:225-227

11. Mitchell DG, Schonholz D, Hilpert PL, et al. Zones of the uterus: discrepancy between US and MR images. Radiology 1990; 174:827-831

12. Levine D, Gosink BB, Johnson LA. Change in endometrial thickness in postmenopausal women undergoing hormone replacement therapy. Radiology 1995;197:603-608

13. Zalud I, Conway C, Shulman H, et al. Endometrial and myometrial thickness and uterine blood flow in postmenopausal women: the influence of hormonal replacement therapy and age. J Ultrasound Med 1993;12:737-741

14. Osmers R, Völksen M, Schauer A. Vaginosonography for early detection of endometrial carcinoma? Lancet 1990;335:15691571

15. Bourne TH. Opinion. Evaluating the endometrium of postmenopausal women with transvaginal ultrasonography. Ultrasound Obstet Gynecol 1995;6:75-80

16. Lev Toaff AS, Toaff ME, Liv JB, et al. Value of sonohysterography in the diagnosis and management of abnormal uterine bleeding. Radiology 1996;201:179-184

17. Goldstein SR. Use of ultrasonohysterography for triage of perimenopausal patients with unexplained uterine bleeding. $\mathrm{Am} \mathrm{J} \mathrm{Ob-}$ stet Gynecol 1994;170:565-570

18. Wood C, Hurley VA, Leoni M. The value of vaginal ultrasound in the management of menorrhagia. Aust NZ J Obstet Gynaecol 1993;33:198-200

19. Dubinsky TJ, Parvey HR, Gormaz G, et al. Transvaginal hysterosonography in the evaluation of small endoluminal masses. $J$ Ultrasound Med 1995;14:1-6

20. Stadtmauer L, Grunfeld L. The significance of endometrial filling defects detected on routine transvaginal sonography. J Ultrasound Med 1995;14:169-172

21. Fukuda M, Shimitzu T, Fukuda K, et al. Transvaginal hysterosonography for differential diagnosis between submucous and intramural myoma. Gynecol Obstet Invest 1993;4:236-239

22. Cicinelli E, Romano F, Anastasio PS, et al. Transabdominal sonohysterography, transvaginal sonography, and hysteroscopy in the evaluation of submucous myomas. Obstet Gynecol 1995;85:42-47

23. Granberg S, Wikland M, Karisson B, et al. Endometrial thickness as measured by endovaginal ultrasonography for identifying endometrial abnormality. Am J Obstet Gynecol 1991;164:47-52

24. Merce LT, Lopez Garcia G, de la Fuente F. Doppler ultrasound assessment of endometrial pathology. Acta Obstet Gynecol Scand 1991;70:525-530

25. Weiner Z, Beck D, Rottem S, et al. Uterine artery flow velocity waveforms and color flow imaging in women with perimenopausal and postmenopausal bleeding. Acta Obstet Gynecol Scand 1993;72:162-166
26. Auslender R, Bornstein J, Dirnfeld M, et al. Vaginal ultrasonography in patients with postmenopausal bleeding. Ultrasound $\mathrm{Ob}$ stet Gynecol 1993;3:426-428

27. Petterson B, Adam H, Lindgren A. Endometrial polyps and hyperplasia as risk factors for endometrial carcinoma. Acta Obstet Gynecol Scand 1985;64:653

28. Karlsson B, Granberg S, Wikland M, et al. Transvaginal ultrasonography of the endometrium in women with postmenopausal bleeding: a nordic multicenter study. Am J Obstet Gynecol 1995;172:1488-1494

29. Cacciatore B, Ramsay T, Lehtovirta P, et al. Transvaginal sonography and hysteroscopy in postmenopausal bleeding. Acta Obstet Gynecol Scand 1994;73:413-416

30. Cohen JR, Luxman D, Sagi J, et al. Sonohysterography for distinguishing endometrial thickening from endometrial polyps in postmenopausal bleeding. Ultrasound Obstet Gynecol 1994; 4:227-230

31. Hulka CA, Hall DA, McCarthy K, et al. Endometrial polyps, hyperplasia, and carcinoma in postmenopausal women: differentiation with endovaginal sonography. Radiology 1994;191:755-758

32. Sheth S, Hamper UM, Kurman RJ. Thickened endometrium in the postmenopausal woman: sonographic-pathologic correlation. Radiology 1993;187:135-139

33. Gimpelson RJ, Rappold HO. A comparative study between panoramic hysteroscopy with directed biopsies and dilatation and curettage: a review of 276 cases. Am J Obstet Gynecol 1988;158:489-492

34. Grimes DA. Diagnostic dilatation and curettage: a reappraisal. Am J Obstet Gynecol 1982;142:1-6

35. Nasri MN, Coast GH. Correlation of ultrasound findings and endometrial histopathology in postmenopausal women. Br J Obstet Gynaecol 1989;96:1333-1338

36. Rudelstorfer R, Nanz S, Bernaschek G. Vaginosonography and its diagnostic value in patients with postmenopausal bleeding. Arch Gynecol Obstet 1990;248:37-44

37. Cacciatore B, Lehtovirta $P$, Wahlström $T$, et al. Contribution of vaginal scanning to sonographic evaluation of endometrial cancer invasion. Acta Oncol 1989;28:585-588

38. Fleischer AC, Dudley BS, Entman SS, et al. Myometrial invasion by endometrial carcinoma: sonographic assessment. Radiology 1987;162:307-310

39. Lehtovirta P, Cacciatore B, Wahlström T, et al. Ultrasonic assessment of endometrial cancer invasion. J Clin Ultrasound 1987; 15:519-524

40. Carlson JA Jr, Arger P, Thompson S, et al. Clinical and pathologic correlation of endometrial cavity fluid detected by ultrasound in the postmenopausal patient. Obstet Gynecol 1991;77:119-122

41. Bourne TH, Lawton F, Leather A, et al. Use of interactive saline instillation and transvaginal ultrasonography to detect tamoxifenassociated endometrial polyps. Ultrasound Obstet Gynecol 1994;4:73-75

42. Gal D, Kopel S, Bahevkin M, et al. Oncogenic potential of tamoxifen on endometria of postmenopausal women with breast cancer: preliminary report. Gynecol Oncol 1991;42:120-123

43. Malfetano JH. Tamoxifen-associated endometrial carcinoma in postmenopausal breast cancer patients. Gynecol Oncol 1990; 39:82-84

44. Perrot N, Guyot B, Antoine M, et al. The effects of tamoxifen on the endometrium. Ultrasound Obstet Gynecol 1994;4:83-84

45. Goldstein SR. Unusual ultrasonographic appearance of the uterus in patients receiving tamoxifen. Am J Obstet Gynecol 1994;170:447-451

46. Hulka CA, Hall DA. Endometrial abnormalities associated with tamoxifen therapy for breast cancer: sonographic and pathologic correlation. AJR 1993;160:809-812

47. Pryse-Davies J. Tamoxifen and the uterus. Ultrasound Obstet Gynecol 1994;4:4-6 